Revue scientifique sur la conception et l'aménagement de l'espace

16 | 2017

Arbres et paysages

\title{
L'arbre urbain : métissage et créolité à Tamatave (Madagascar)
}

The Urban Tree: Cross-Cultural Influences and Creole Identity in Toamasina (Madagascar)

\section{Jean-Baptiste Bing}

\section{(2) OpenEdition} Journals

Édition électronique

URL : http://journals.openedition.org/paysage/5004

DOI : 10.4000/paysage.5004

ISSN : 1969-6124

Éditeur :

École nationale supérieure du paysage de Versailles-Marseille, Institut national des sciences appliquées Centre Val de Loire - École de la nature et du paysage, École nationale supérieure d'architecture et de paysage de Bordeaux, École nationale supérieure d'architecture et de paysage de Lille, Agrocampus Angers

\section{Référence électronique}

Jean-Baptiste Bing, «L'arbre urbain : métissage et créolité à Tamatave (Madagascar) », Projets de paysage [En ligne], 16 | 2017, mis en ligne le 01 juillet 2017, consulté le 30 avril 2020. URL : http:// journals.openedition.org/paysage/5004; DOI : https://doi.org/10.4000/paysage.5004

Ce document a été généré automatiquement le 30 avril 2020.

Projets de paysage 


\title{
L'arbre urbain : métissage et créolité à Tamatave (Madagascar)
}

\author{
The Urban Tree: Cross-Cultural Influences and Creole Identity in Toamasina \\ (Madagascar)
}

Jean-Baptiste Bing

1 Comptant plus de 300000 habitants, Tamatave est (après la capitale Antananarivo) la deuxième plus grosse agglomération de Madagascar. En retrait par rapport à d'autres cités de la côte betsimisaraka à l'époque des royaumes locaux (xvII $\mathrm{e}$-XVIII ${ }^{\mathrm{e}}$ siècles), la ville a commencé à prendre de l'importance sous le règne du roi merina Radama $I^{\text {er }}$ (1816-1828, considéré avec son père Andrianampoinimerina comme l'un des unificateurs de l'île) grâce à son port qui, depuis, est devenu le plus important du pays. Ce rôle d'interface entre l'extérieur et l'autorité souveraine (royaume merina au xIX siècle, pouvoir colonial de 1895 à 1960, république indépendante depuis), de vitrine à destination des visiteurs, et enfin son ouverture sur l'océan Indien ont donné à Tamatave un caractère métis dont la créolité est une des multiples déclinaisons, particulièrement visible dans le paysage. Cet article interroge la place et les usages de l'arbre dans la construction de ce métissage paysager urbain et notamment de sa part créole.

2 Il s'appuie sur un séjour de deux années à Tamatave: formateur d'enseignants en langue française et chargé d'organiser les activités culturelles du centre de formation, j'ai pu pratiquer assez largement l'observation participative, tant auprès des collègues et des élèves-enseignants que auprès d'autres habitants (partenaires professionnels, voisins, amis...) issus de toutes les catégories sociales, depuis l'élite culturelle jusqu'aux ruraux en exil. Flânerie urbaine en solitaire, parcours commentés souvent par des habitants et plusieurs fois par une guide, entretiens formels et informels avec des proches et de simples connaissances : tout cela a débouché sur des photographies et sur des carnets de notes. Prises en général après coup, ces notes concernaient donc des sujets variés et n'avaient pas d'objet de recherche; leur compilation et leur confrontation à d'autres types de données (blogs, articles de presse, pétitions...) permettent néanmoins de dégager certaines tendances ${ }^{1}$. Si l'analyse porte 
essentiellement sur les trois quartiers de la ville les plus fréquentés au cours du séjour (Tanamakoa, Analakinina et le centre-ville, dont on distinguera Ampasimazava - où s'élevait la ville précoloniale - et le reste du centre - qui accueillit les bâtiments administratifs de la colonie), elle s'efforce de ne pas ignorer pour autant le reste de l'agglomération.

La première partie dégagera les éléments théoriques et historiques qui préciseront le lien du paysage urbain tamatavien au métissage et à la créolité de la ville ; la deuxième examinera les différentes fonctions de l'arbre dans ce paysage, les rattachera aux territorialités variées qui s'y expriment et aboutira à quelques pistes de réflexion.

Figure 1. Carte de Tamatave

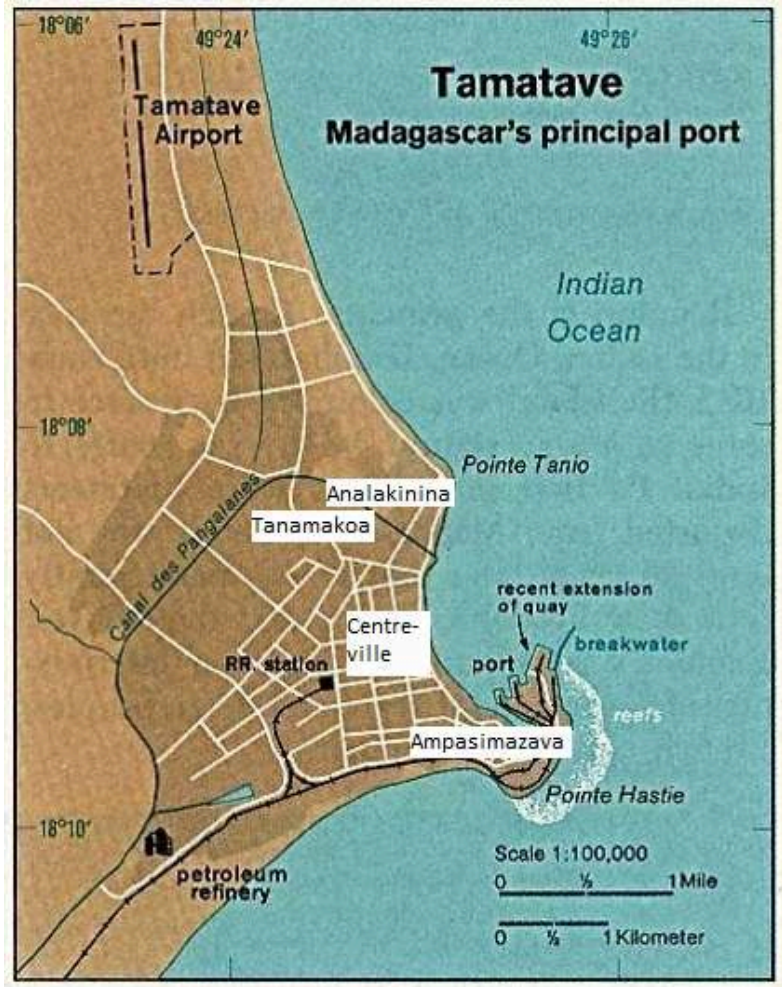

Réalisation : Jean-Baptiste Bing, février 2017, d'après http://www.philadelphiaproject.co.za/images/ maps/mad06.jpg.

\section{Le paysage tamatavien, « empreinte et matrice » du métissage urbain}

4 Le caractère métis de la ville et le paysage urbain entretiennent une rétroaction réciproque et positive, un rapport d'« empreinte et matrice» (Berque, 2009). D'où un double corollaire, a priori contradictoire mais qui, en fait, constitue les deux faces d'une même pièce : primo, si le paysage urbain ne peut être abstrait de l'ouverture ancienne de la ville aux étrangers et de la diversité culturelle de ses habitants, il a sa propre dynamique, son propre rythme, et répond à des évolutions qui lui sont propres ; secundo, bien que nous puissions distinguer analytiquement ces deux phénomènes, ils sont, au quotidien, intriqués l'un l'autre et contemporains. 


\section{Tamatave, métisse ou créole ?}

5 Tamatave, à l'instar de Majunga qui lui a longtemps disputé le titre de premier port de l'île, est une ville qui peut facilement être qualifiée de «métissée " ou de «multiculturelle ${ }^{2}$ (Men et al., 2003) : aux Malgaches originaires de toutes les « ethnies ${ }^{3}$ » venus chercher - entre autres - une économie plus dynamique que chez eux et/ou faire jouer les réseaux familiaux et villageois s'ajoutent, d'une part, les Asiatiques (d'origine chinoise et indienne ${ }^{4}$ et généralement de nationalité malgache) et, d'autre part, des vazaha ("Blancs », «Occidentaux ») en grande majorité étrangers ${ }^{5}$. Cela induit une double spécificité de la ville, d'une part, par rapport à l'arrière-pays betsimisaraka (moins métissé et moins créole), d'autre part, par rapport à la capitale Antananarivo (moins créole, moins " océanindienne »). Les habitants reconnaissent assez largement ce caractère métis, que ce soit pour le revendiquer, le déplorer ou simplement le constater : la première attitude, minoritaire, est généralement le fait d'habitants eux-mêmes métis issus d'ancêtres chinois ou européens; la deuxième, encore plus rare, consiste pour des Betsimisaraka à exprimer un malaise du fait qu'ils ne se sentent pas en pays betsimisaraka à Tamatave bien que celle-ci se situe en plein cœur de celui-là et soit souvent considérée comme sa « capitale »; la troisième demeure la plus courante ${ }^{6} .$.

Ce caractère métis se double d'une certaine créolité puisant son origine dans les Mascareignes voisines avec lesquelles, par-delà les ruptures coloniales et postcoloniales, Tamatave et la côte est entretiennent des relations serrées depuis plusieurs siècles. Insistons sur ce point : cette créolité, relative, ne fait pas de Tamatave une ville proprement créole comme peuvent l'être les îles de la Réunion ou Maurice. Aux yeux des Malgaches, les Créoles en tant que communauté ont disparu avec la colonisation : comme les Vazaha, ceux qui vivent à Tamatave en provenance des îles voisines sont généralement de passage, et les rares ayant fait souche depuis l'Indépendance sont désormais considérés comme des zanatany ${ }^{7}$ et/ou confondus avec les métis (Bavoux, 1990, p. 16-21). Dans cette a-créolité, il y a deux exceptions, l'une parfois éphémère, l'autre plus profonde : la première est constituée des restaurants s'affichant comme créoles (quelques dizaines de personnes au plus, mais très visibles) ; la seconde est la petite communauté sourti, composée de quelques centaines de personnes et dont les trois piliers identitaires sont une lointaine origine gujarati (ibid., p. 29-30), l'islam sunnite (ibid., p. 31-33) et une langue créole issue des Mascareignes (ibid., p. 43-75).

7 La créolité est donc l'un des aspects pris par le caractère métis de Tamatave, mais celuici déborde largement celle-là. Étant donné la faiblesse numérique des Créoles et des créolophones, la créolité tamatavienne ne subsiste quasiment plus que dans le paysage et notamment, nous le verrons, dans le bâti. Plus général, le métissage est aussi moins facilement identifiable tant il imprègne la population et le paysage urbain dans tous ses aspects.

\section{Premières prises : le bâti et le panorama}

Pour saisir en détail ce double processus (métissage/créolisation) et son expression paysagère, prenons comme première entrée - comme première prise $e^{8}-$ le bâti. Aucune construction, dans son architecture contemporaine, n'est antérieure à la colonisation. De très nombreux bâtiments du centre-ville (officiels, publics ou privés) relèvent d'une 
architecture créole, du type que l'on retrouve à la Réunion, à Maurice ou aux Seychelles 9 . Traditionnellement il s'agit de maisons en bois, comportant une varangue, un toit très pentu et (souvent mais pas toujours) plusieurs étages ; institution culturelle majeure de la ville, l'Alliance française est installée dans une maison de ce type (figure 2), de même que le consulat britannique. Concernant les bâtiments, donc, l'arbre n'entre dans la composition du métissage tamatavien - notamment de sa composante créole - qu'abattu et débité, à titre de matériau de construction. Ce rôle apparaît d'autant plus limité que la plupart des maisons «créoles » de Tamatave sont aujourd'hui en béton : plus moderne, permettant des constructions plus vastes tout en conservant les éléments caractéristiques de la créolité (souvent la varangue et les étages, plus rarement la pente du toit), ce matériau est plus prestigieux.

Figure 2. Vue de l'Alliance française de Tamatave

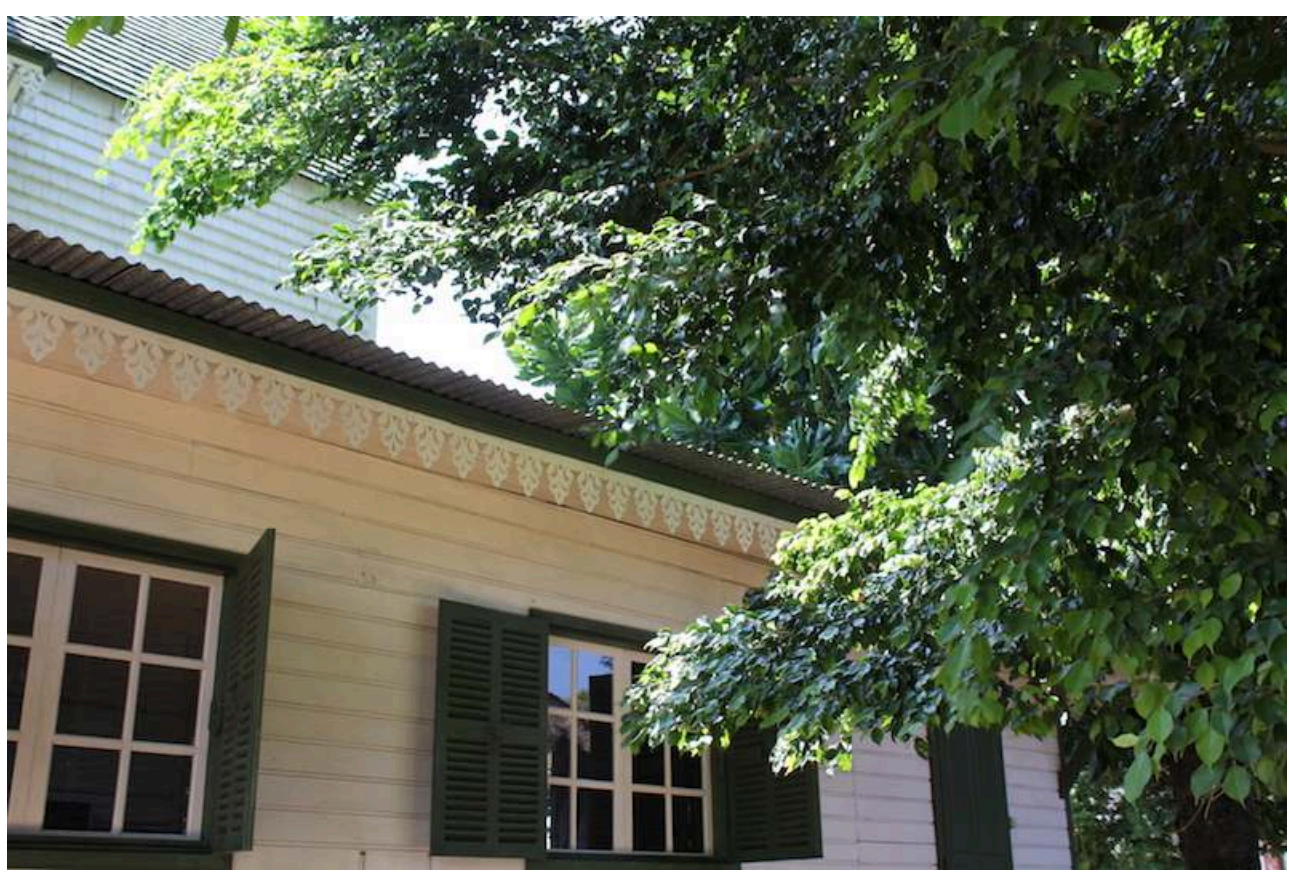

Maison créole dite «Villa Bang » (du nom de ses anciens propriétaires issus de la diaspora norvégienne de Madagascar). La varangue, qui subsiste à l'avant de la maison, a été couverte sur le côté pour fournir deux salles de classe ; les étages abritent les bureaux.

Source : Ludovic Pagniez, septembre 2010.

9 Cependant le paysage urbain ne se limite pas au bâti. Or, le sud du centre-ville, où s'élevait la ville précoloniale, a conservé de cette époque d'abord son nom (Ampasimazava), et surtout certaines places qui, en raison de leur ancien statut et bien que situées en pleine ville, sont demeurées vierges de construction. Nous aborderons le détail de leur histoire et des enjeux qui s'y nouent ci-dessous. Signalons ici que, du strict point de vue de la forme, leur caractère arboré les singularise dans le paysage urbain: on voit sur la figure 3 que, vue depuis le front de mer, Ampasimazava est littéralement dominée par les banians de la place Bienaimé; depuis la mer, avec les infrastructures portuaires, ces arbres constituent même le principal amer de Tamatave. 
Figure 3. Ampasimazava vue depuis le front de mer

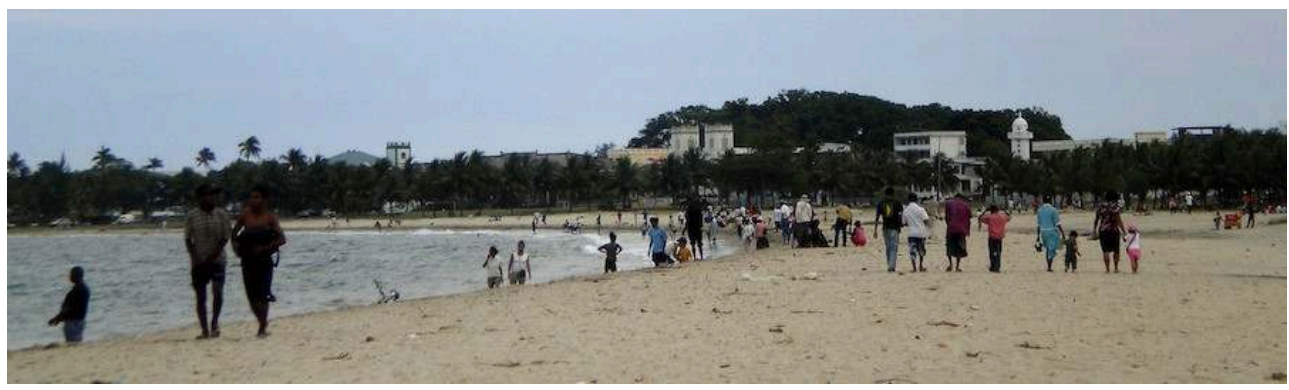

De gauche à droite, se détachent la tour unique d'un ancien comptoir colonial devenu la Bank of Africa, le double clocher d'une église, un immeuble, le minaret d'une mosquée - et surtout, la canopée qui domine la place Bienaimé.

Source : Ruli Bing, novembre 2009.

10 Cette histoire, bien entendu, se poursuit. Si nous conservons la focale sur le bâti, l'alternative bois/béton est loin d'avoir été tranchée, même en se limitant aux quartiers du centre-ville occupés surtout par les élites sociales ${ }^{10}$. En effet dans certains milieux expatriés, le bois, matériau vintage, redevient très tendance ; il n'est pas impossible que, dans un proche avenir, cette mode se diffuse à l'élite culturelle malgache (cf. Bing 2012 à propos des liens élite locale/expatriés concernant la langue). Ce n'est certes pas encore le cas, mais des raisons très concrètes en rendent la perspective réaliste : le paysage ordinaire se vit de manière quotidienne (Sansot, 1983) et multisensorielle (Ernwein et Sgard, 2012) et, à Tamatave, l'alliance de chaleur et d'humidité qui caractérise le climat local peut devenir éprouvant y compris pour les Malgaches (surtout ceux venus des hautes terres). Or le béton, qui absorbe plus la chaleur du jour, entraîne une utilisation souvent accrue de la climatisation alors même que la fourniture en électricité connaît régulièrement des coupures de courant parfois longues; le bois, lui, laisse mieux passer la fraîcheur. D'où le choix, par certains expatriés, de maisons en bois et en toit de chaume (figure 4). Si, dans un proche avenir, sont importées à Madagascar les techniques - qui, actuellement, s'affirment en Asie tropicale - permettant de construire en bois des bâtiments de grande taille, alors le bois pourrait remplacer le béton comme matériau de confort et de prestige aux yeux de l'élite locale. 
Figure 4. Maison en bois avec toiture de falafala (chaume à base de palmes d'arbre du voyageur), sise dans le quartier d'Analakinina

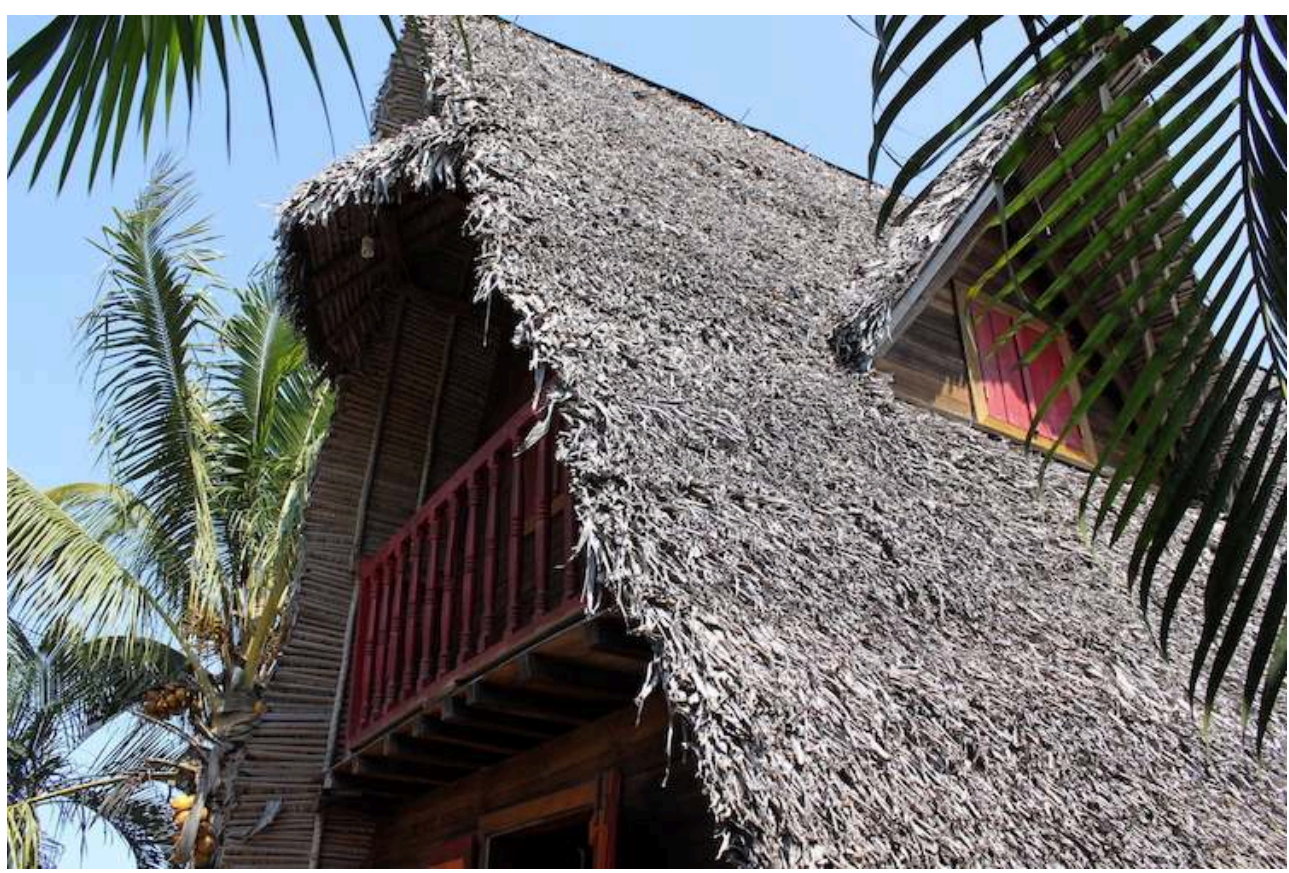

Occupée par des expatriés (aux revenus certes plus modestes que la moyenne de ce groupe), elle témoigne - avec les maisons en béton qui parsèment les environs en peau de léopard - de la mixité sociale de certains quartiers (Analakinina, Tanamakoa...) de la première couronne entourant le centreville.

Source : Ludovic Pagniez, septembre 2010.

\section{L'arbre multifonctionnel}

11 Nous n'en sommes pas encore là. De plus, bien que la ville forme bel et bien une unité qu'il est possible, à l'échelle régionale, de distinguer d'un arrière-pays rural, elle n'est pas pour autant homogène. À une échelle plus locale, elle est le cadre où cohabitent des territorialités variées, prises dans des rapports de pouvoir, qui peuvent s'ignorer, coopérer, s'affronter; elle est aussi le produit de cette coexistence. On retrouve là la logique complexe du double caractère $d^{\prime}$ ' empreinte et matrice »: le système global (la ville) permet le jeu de composantes, mais il est aussi déterminé par lui (Péguy, 2001). Il est donc nécessaire, pour mieux saisir la place de l'arbre dans le processus de métissage dont est issu le paysage urbain actuel, de trouver d'autres prises permettant de saisir la complexité du paysage urbain. Pour cela nous nous pencherons sur les différents usages de l'arbre, en distinguant, d'une part, l'arbre monumental et, d'autre part, l'arbre convivial. Il va de soi que ces deux fonctions ne sont pas exclusives : un même arbre peut être à la fois monumental et convivial (cf. ci-dessous le cas de la place Bienaimé). Cependant, selon qu'on le considère sous un angle ou sous l'autre, il devient le lieu où se déploient des territorialités vécues par des acteurs variés. C'est cette multiplicité des usages du paysage arboré que nous nous allons maintenant décrypter, à travers les sens qu'elle porte, les actions qu'elle permet et les conflits qu'elle engendre. 


\section{L'arbre monumental : un matériau vivant au service de l'urbanisme}

12 planifiées et organisées par les autorités. En tant que monument, l'arbre est un élément des politiques publiques d'urbanisme, résolument inscrit dans une durée pluridécennale, voire séculaire. Depuis les débuts de la colonisation (1895) jusqu'à aujourd'hui, les autorités locales et nationales en charge de la ville ont largement exploité l'arbre comme élément du paysage urbain. Le plan du centre-ville tel que conçu et organisé par le colonisateur, très largement orthogonal, prévoyait de doter les principales avenues d'arbres d'ornementation, sur le modèle de ce qui se faisait en métropole. Il n'y a globalement pas eu de rupture avec ce passé : les grandes artères associent toujours une toponymie largement héritée de l'époque coloniale (le «boulevard Ratsimilaho » apparaît bien isolé face à l'« avenue Maréchal-Joffre », la «rue Grandidier» et autres «boulevard de la Libération »...) à des arbres dont les essences sont aujourd'hui très présentes dans toutes les villes du pays, qu'elles soient locales (flamboyant: Delonix regia) ou d'origine exotique (jacaranda: Jacaranda mimosifolia).

La répétition de ce modèle dans d'autres villes contribue d'ailleurs à donner une unité de style à l'urbanisme national: la monumentale avenue, qui va du front de mer à l'hôtel de ville ${ }^{11}$ via un terre-plein central planté de palmiers-bouteille (Hyophorbe verschaffeltii : figure 5), se retrouve par exemple en plus petit à Fénérive-Est (capitale de la région Analanijirofo). Certes, en procédant ainsi, les autorités françaises ne visaient pas à promouvoir le caractère métis de la ville en tant que tel : l'idéologie coloniale aurait plutôt eu tendance à ignorer ce mélange identitaire fluctuant et peu respectueux des hiérarchies établies, voire à s'en méfier ; il s'agissait plutôt d'adapter une pratique européenne (larges avenues ornées d'arbres) aux conditions locales (climat tropical humide, sol sablonneux), et d'ancrer dans le quotidien des habitants cette traduction paysagère de l'unité et de l'indivisibilité de la "plus grande France ». Par la suite, les régimes qui se sont succédé depuis l'Indépendance cherchèrent à se légitimer à la fois comme héritiers de l'unité nationale précoloniale et comme capables d'assumer la modernité; ils récupérèrent donc ces formes urbaines en leur injectant un sens nouveau, rebaptisant par exemple "avenue de l'Indépendance" les artères ciévoquées, monumentalisées par leur terre-plein planté. Ce faisant ils valorisèrent (et valorisent encore) d'un seul tenant une autochtonie réifiée et la modernité technocratique; par contraste, plus fluctuant et spontané, le métissage apparaît là encore comme un foyer possible d'opposition politique, voire ethnique, qu'il convient de neutraliser à défaut de réussir à le manipuler comme peut l'être l'ethnicité (Bing, 2012, 2014). 
Figure 5. L'avenue de l'Indépendance décorée après la fête nationale

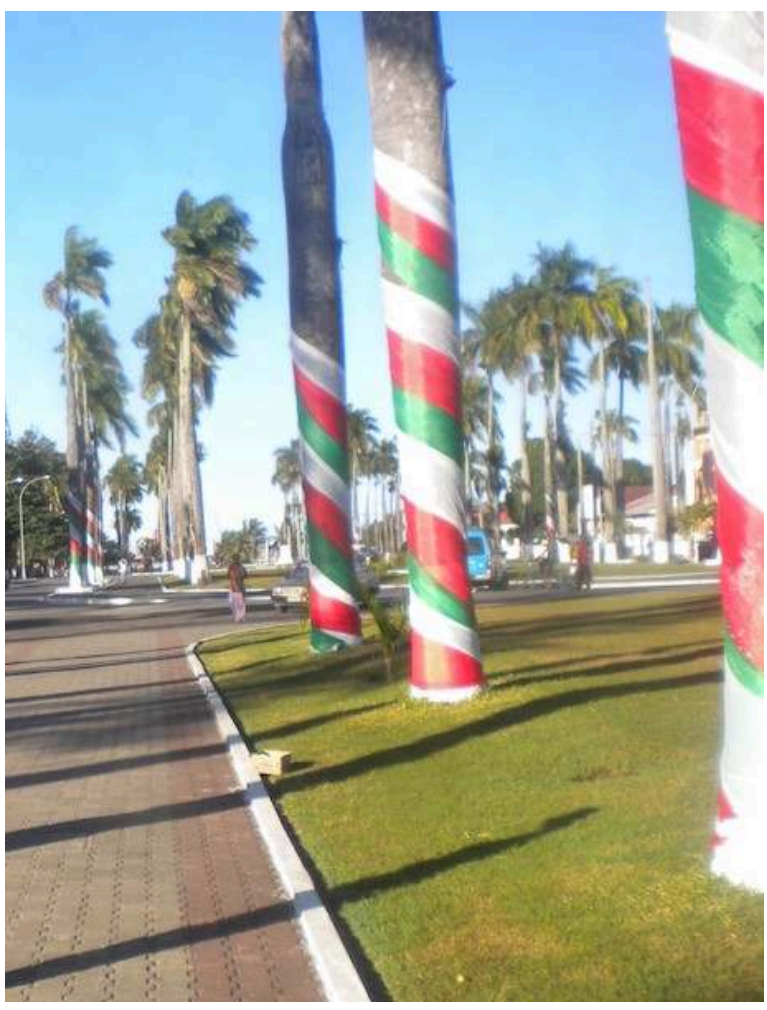

Ancien champ de course, elle a été transformée en artère reliant le front de mer à l'hôtel de ville. Ouverte aux voitures toute l'année, elle sert de cadre aux grandes cérémonies publiques.

Source : Ruli Bing, juillet 2011

C'est aussi dans ce cadre d'assimilation et de neutralisation qu'il faut placer l'histoire des quelques éléments précoloniaux d'Ampasimazava : si certains aspects urbains y ont été conservés, ils ont été chargés de significations et de fonctions nouvelles. Un cas est particulièrement intéressant quant au rôle joué par les arbres: le doublon place du Marché/place de la Colonne. Avant la colonisation, occupaient ces emplacements un cimetière et - la toponymie parle d'elle-même - un marché. Pour des raisons d'hygiène, les autorités françaises ont rapidement décidé de déplacer ces institutions hors d'une ville en pleine croissance, et d'y établir des espaces verts ${ }^{12}$. De nos jours, ces lieux sont devenus complètement insalubres : non entretenus par les autorités municipales, ils ont été saccagés par les routiers qui y parquent leurs camions (figure 6). Depuis plusieurs années un promoteur veut y implanter un centre commercial avec, semble-til, un soutien tacite de la mairie qui voit d'un bon œil tout ce qui peut apporter de la croissance économique et rendre la ville plus salubre sans qu'elle n'ait à débourser un sou. Face à ce projet (si opaque que, tantôt, la place concernée est celle de la Colonne et, tantôt, celle du Marché...) une opposition s'est faite jour, unissant les habitants du pourtour des deux places (pauvres, ils squattent des bâtiments dont ils ne sont pas propriétaires et craignent l'expulsion) et des militants pour la protection du patrimoine tamatavien (plutôt issus de l'élite). 
Figure 6. Aux abords de la place de la Colonne, un camion hors d'usage abandonné sur place avec ses containers, transformé en gargote informelle

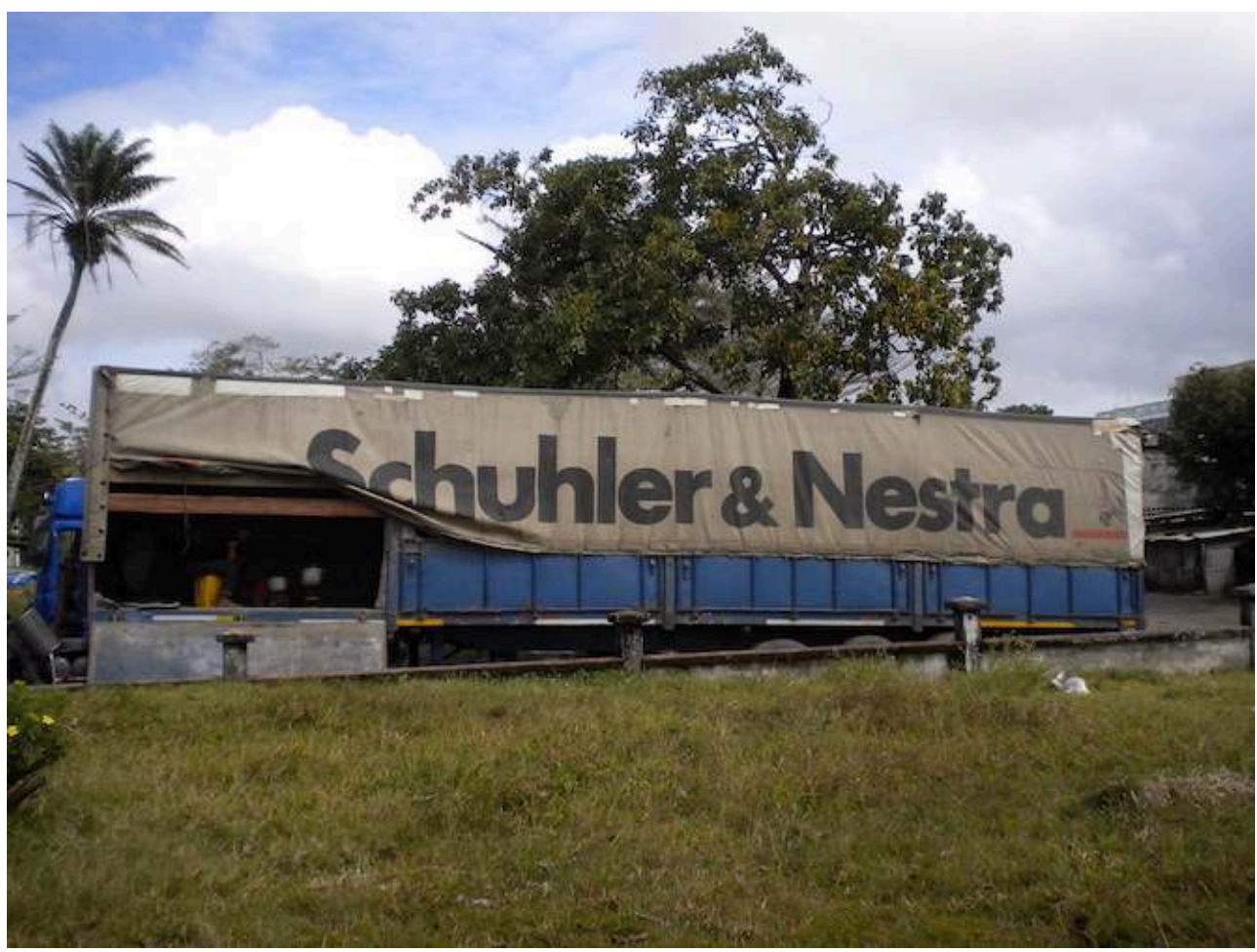

Photo : Jean-Baptiste Bing, août 2010.

Un arbre sis place du Marché joue, pour ces opposants, un rôle de symbole: un flamboyant abattu, qui aurait "saigné » lors de sa coupe et dont les morceaux sont pour cela considérés comme fady (sacrés, tabous) et laissés sur place. Certains assurent qu'il continue de fleurir, voire aurait développé de nouvelles racines... Ce déplacement de la controverse sur le terrain sacré est d'autant moins surprenant que son ancienne fonction de cimetière continue de sacraliser la place de la Colonne, le culte des ancêtres étant un trait caractéristique de la culture malgache. Apparaît ici une limite de la dichotomie proposée entre arbre monumental et arbre convivial : un même arbre peut être à la fois monument et protection, de même que la fonction décorative n'exclut pas la fonction écologique ; cependant, selon les acteurs et les enjeux, l'accent sera mis sur tel ou tel aspect. Dans le cas de la place du Marché, la monumentalité et la sacralité sont aujourd'hui mises en avant par les habitants et les associatifs, face aux pouvoirs politique et économique qui souhaiteraient n'y voir que du matériel dénué de valeur alors que, à l'origine, c'est ce pouvoir politique qui, avec cet aménagement jardinier, avait imposé sa marque sur la monumentalité du lieu.

Autre cas, déjà évoqué ci-dessus : la place Bienaimé. Ancien espace sacré lié à la royauté locale et aux rituels de divination, elle est devenue à l'époque française un lieu de parcage des immigrants asiatiques puis, assez vite, un lieu de promenade et de loisir ; aujourd'hui elle sert notamment de terrains de pétanque ${ }^{13}$. À l'orée du $\mathrm{xx}^{\mathrm{e}}$ siècle, les autorités coloniales ont planté son pourtour de douze banians (Ficus benghalensis) dont les semences ont été importées d'Inde. Désormais géants, ils ombragent toute la place (figure 7) et dominent la ville (figure 3). Les banians n'ont, à Madagascar, aucune connotation sacrée comme en Inde, à Java ou dans le Pacifique ; ils ne suffisent donc pas 
pour perpétuer le caractère sacré du lieu. Un sacrifice de zébu y a donc été organisé, à l'issue duquel un bucrane a été planté sur l'un des banians (figure 8$)^{14}$.

Figure 7. La place Bienaimé, dominée par ses douze banians

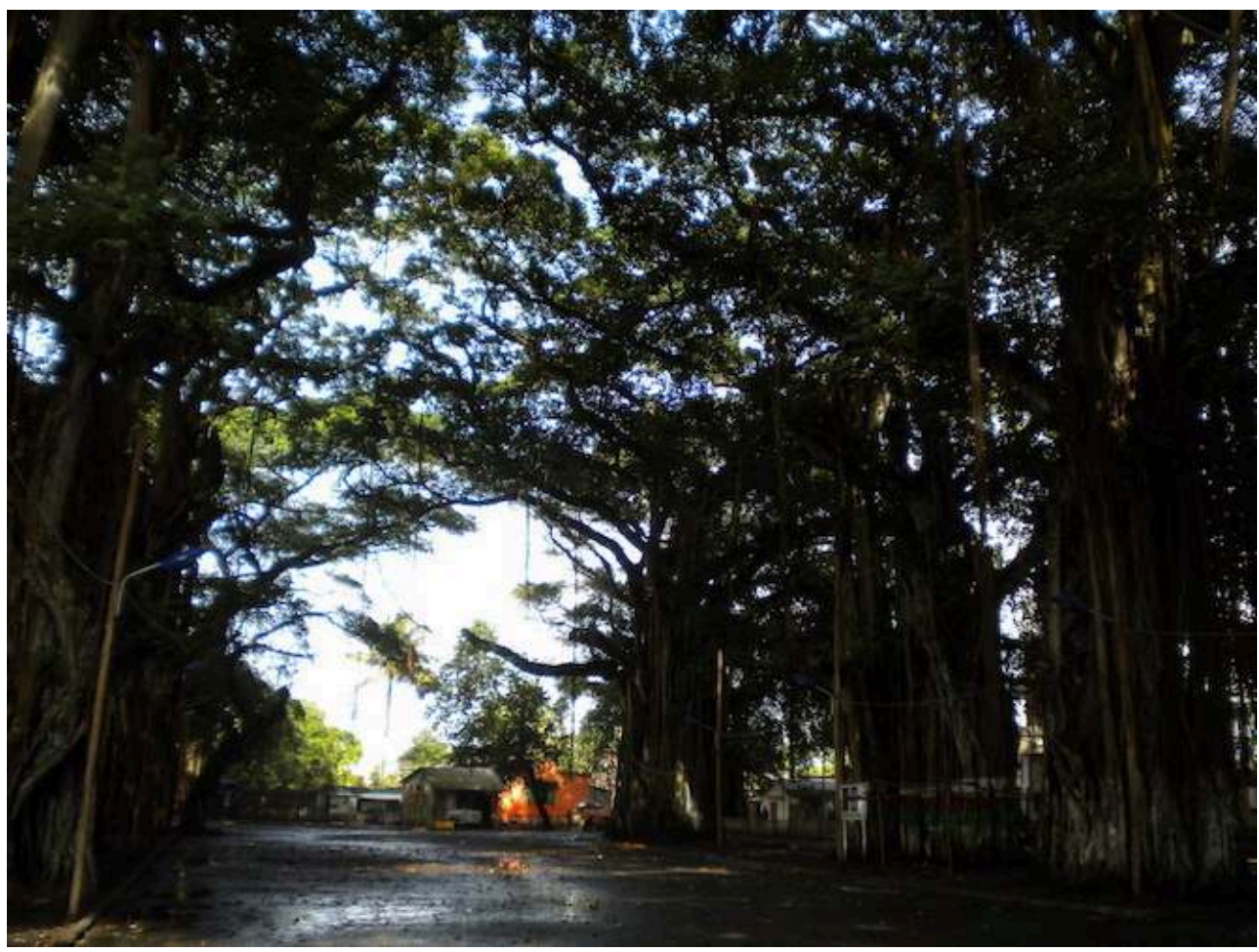

Source : Jean-Baptiste Bing, août 2010. 
Figure 8. Bucrane implanté lors d'un sacrifice de zébu

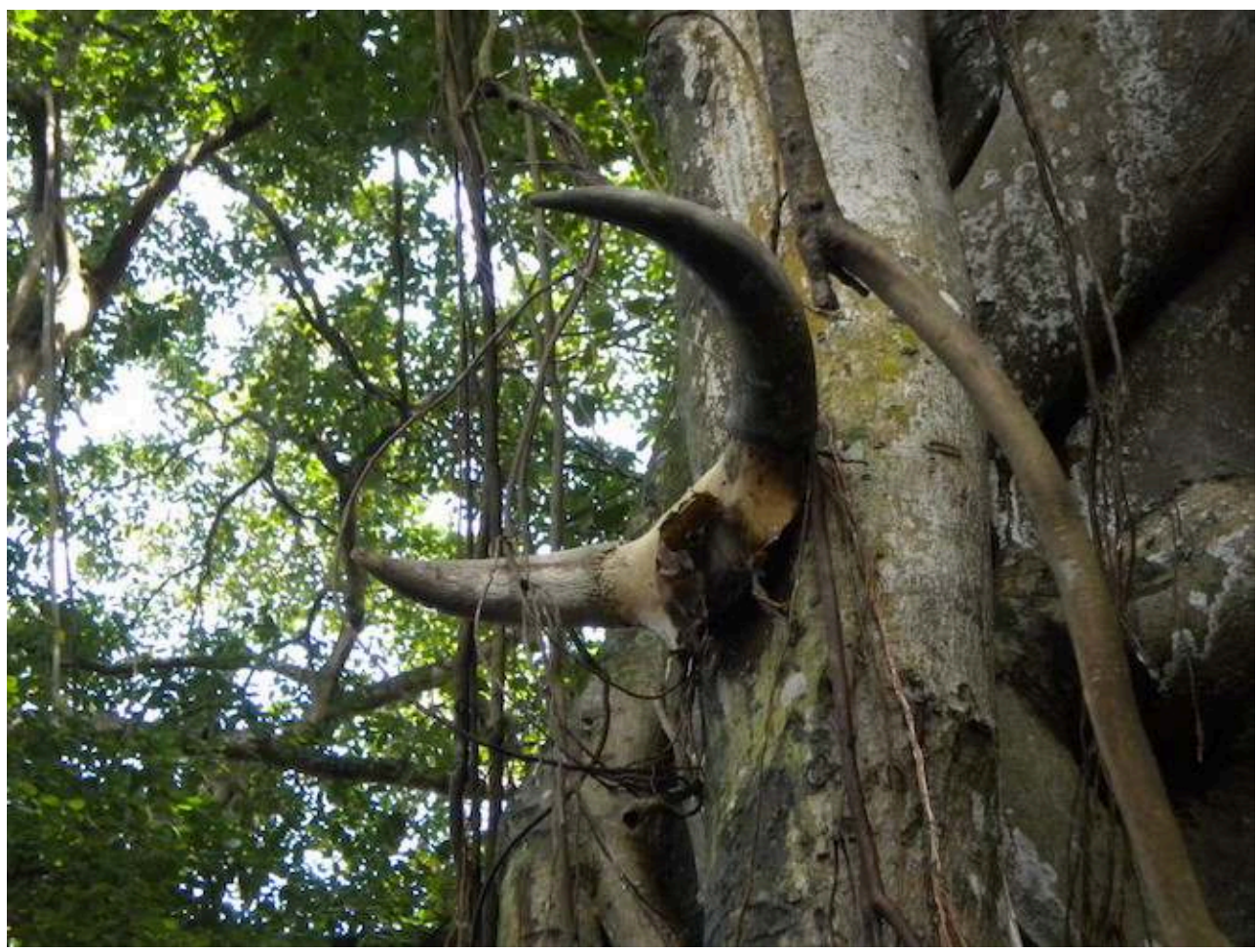

Source : Jean-Baptiste Bing, août 2010.

17 Dans le cas des places Bienaimé, de la Colonne et du Marché, l'arbre a donc servi aux différentes autorités à organiser le métissage urbain, non pas en le recherchant en tant que tel mais afin de consolider leur pouvoir et d'implanter leur marque sur les lieux. Face à ces efforts, la perpétuation des anciennes territorialités s'est faite grâce à une réappropriation des lieux par les habitants, d'une part, et, d'autre part, grâce à une série de coïncidences qui montrent bien la part de contingence qui existe en matière paysagère (Berque, 2008, 2009). Ainsi, dans un souci d'aménagement rationnel, les banians de la place Bienaimé ont été plantés aux points cardinaux ; à leur insu, ils ont ainsi respecté une caractéristique fondamentale du système malgache de divination et d'astrologie qui a marqué le lieu dans le passé précolonial. (De plus, et là encore le hasard a bien fait les choses, il y a une certaine proximité entre le nom de Timasy Vavitiana, la principale "prophétesse » ayant officié sur place, et celui de l'actuel éponyme M. Bienaimé.)

Si la fonction de monumentalité des arbres du centre-ville ne fait pas de doute, en revanche il faut relativiser la notion de planification qui semble naturellement l'accompagner: on l'a dit, les autorités municipales ont assez largement délaissé la gestion des espaces verts. Nous en verrons l'essentiel des conséquences, en termes d'écologie urbaine, ci-dessous; contentons-nous pour l'instant de relever que, en termes strictement paysagers, l'entretien des arbres propriété municipale se limite surtout à des tailles radicales, qui mettent à mal d'autres fonctions publiques des arbres (ombrage des rues...) ainsi que, si elles se répètent trop souvent, leur longévité. Or, cette convivialité portée par les arbres est essentielle: dans le cas des conflits autour des places de la Colonne et du Marché, les arbres servent de médiateurs entre des revendications contradictoires, voire incompatibles; aux yeux de la municipalité la 
fonction monumentale ne justifie plus la présence des arbres, tandis que pour les habitants et les militants du patrimoine les fonctions protectrice et mémorielle conservent toute leur force.

\section{L'arbre convivial : une sylviculture urbaine, palliatif des insuffisances politiques?}

L'exemple des trois places d'Ampasimazava nous a ouvert la porte des autres niveaux de territorialités: vernaculaires, «des échanges» et sacrées. Penchons-nous maintenant surtout sur les premières, et sur l'arbre outil de convivialité, c'est-à-dire permettant aux habitants de maîtriser la ville (Illich, 1973). Cela nous conduira par-delà le centre-ville, dans d'autres quartiers de Tamatave: nous nous intéresserons surtout ici aux quartiers de Tanamakoa et Analakininy, situés au nord du centre-ville et qui ont été les plus fréquentés durant nos deux années de séjour, mais les incursions faites dans les autres quartiers de la ville incitent à penser que la situation y est semblable. Interface entre la périphérie récemment urbanisée et le centre-ville, ces quartiers sont d'une urbanisation relativement ancienne; en tout cas la toponymie témoigne de leur ancienne intégration dans un hinterland au service de la ville, ainsi que d'un métissage imposé par les circonstances: Analakinina («forêt de quinine ») désigne une ancienne plantation qui fournissait un produit indispensable à l'élite coloniale; quant à Tanamakoa, il s'agit du quartier réservé aux «Makoa», supplétifs africains de la colonisation qui, à l'instar des Créoles (Bavoux, 1990), ont laissé un assez mauvais souvenir à Madagascar (Alexandre, 2007).

20 Si le centre a été largement planifié par les autorités, les quartiers qui l'entourent mélangent habitats formel et informel. En dehors des grandes artères (avenue de la Bourdonnais...), le plan est composé de ruelles anonymes et tortueuses dont peu sont assez larges pour laisser circuler une voiture (figure 9). La population y est socialement très mixte : implantées de-ci de là parmi celles en bois, les maisons en béton ne sont pas rares et symbolisent la réussite sociale des propriétaires; certains pâtés de maison offrent même de notables exemples de gentrification tantôt spontanée, tantôt résultant d'opérations de promotion immobilière. L'immense majorité des bâtisses est toutefois construite sur le modèle de la case rurale du pays betsimisaraka, avec les moyens du bord et en employant des matériaux courants, peu chers et de provenance proche : bois peu onéreux pour le plancher et les murs (bambous tressés aussi, plus rarement), palmes de ravenala (Ravenala madagascariensis souvent dit " arbre du voyageur ») pour la toiture ( toits en falafala »).

21 Dans ces deux quartiers les arbres ne sont pas moins rares qu'au centre-ville; et leur fonction est fort différente. Dans le centre les arbres sont essentiellement décoratifs, qu'ils ornent les rues ou les jardins privés. À Tanamakoa et Analakinina, ces arbres sont rarement plantés dans les voies de passage: tracées au fil d'une urbanisation assez largement spontanée, souvent tortueuses, celles-ci n'offrent en effet pas assez de place. Concentrés dans des jardins souvent de petite taille, les arbres ont, outre une valeur décorative et affective qui peut unir les propriétaires au lieu (Tuan, 2006 ; Bing, 2015b), une visée utilitaire: ils ombragent les jardinets entourant les maisons; souvent fruitiers (manguiers, papayers...), ils fournissent de la matière première à la fois à la consommation domestique et à de petits emplois informels (vendeurs de rue de jus de tamarin...); enfin, même si l'essentiel du bois de construction provient des zones 
rurales extérieures à la ville, ces arbres peuvent apporter un complément ponctuel. Cette diversité des espèces relève aussi d'un processus de métissage, si l'on considère celui-ci comme une malgachisation d'éléments autrefois exotiques: métissage biologique puisque, en dehors de l'arbre du voyageur, toutes ces essences furent à l'origine importées ; métissage des pratiques aussi, dont un exemple éloquent est fourni par le ramboutan. Originaire d'Asie tropicale, Nephelium lappaceum est fréquemment cultivé, en ville comme à la campagne, et ses fruits se trouvent facilement sur les marchés de Tamatave ; en brousse, ils sont vite coupés sur l'arbre et consommés avec les invités. Contrairement à la vanille, fréquemment cultivée dans les collines de l'Est mais destinée à l'exportation, le ramboutan est donc entré dans le quotidien des habitants; cependant son nom vernaculaire local («litchi chinois») conserve le souvenir de son origine est-asiatique ${ }^{15}$. Du point de vue visuel, ces arbres marquent d'autant plus le paysage qu'ils s'élèvent en général largement au-dessus des maisons ; cependant, et même s'il est des exceptions (souvent en bordure des grandes avenues), ils ont néanmoins souvent une stature moins imposante que ceux du centre-ville - il est vrai que c'est aussi le cas des maisons (figures 9 et 10)...

Figure 9. Dans le quartier de Tanamakoa, ruelle durant la saison des pluies

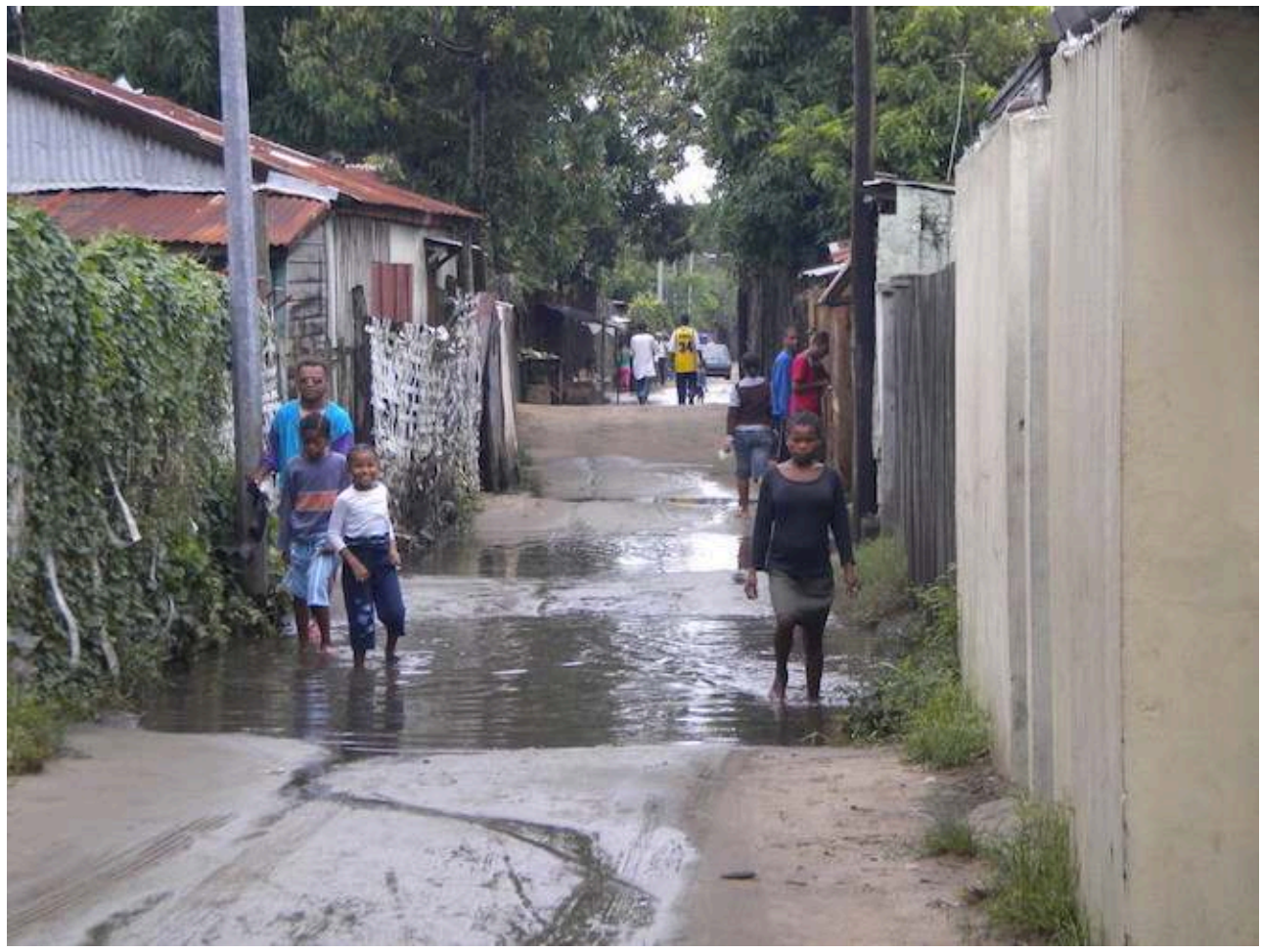

Source : Ruli Bing, janvier 2010. 


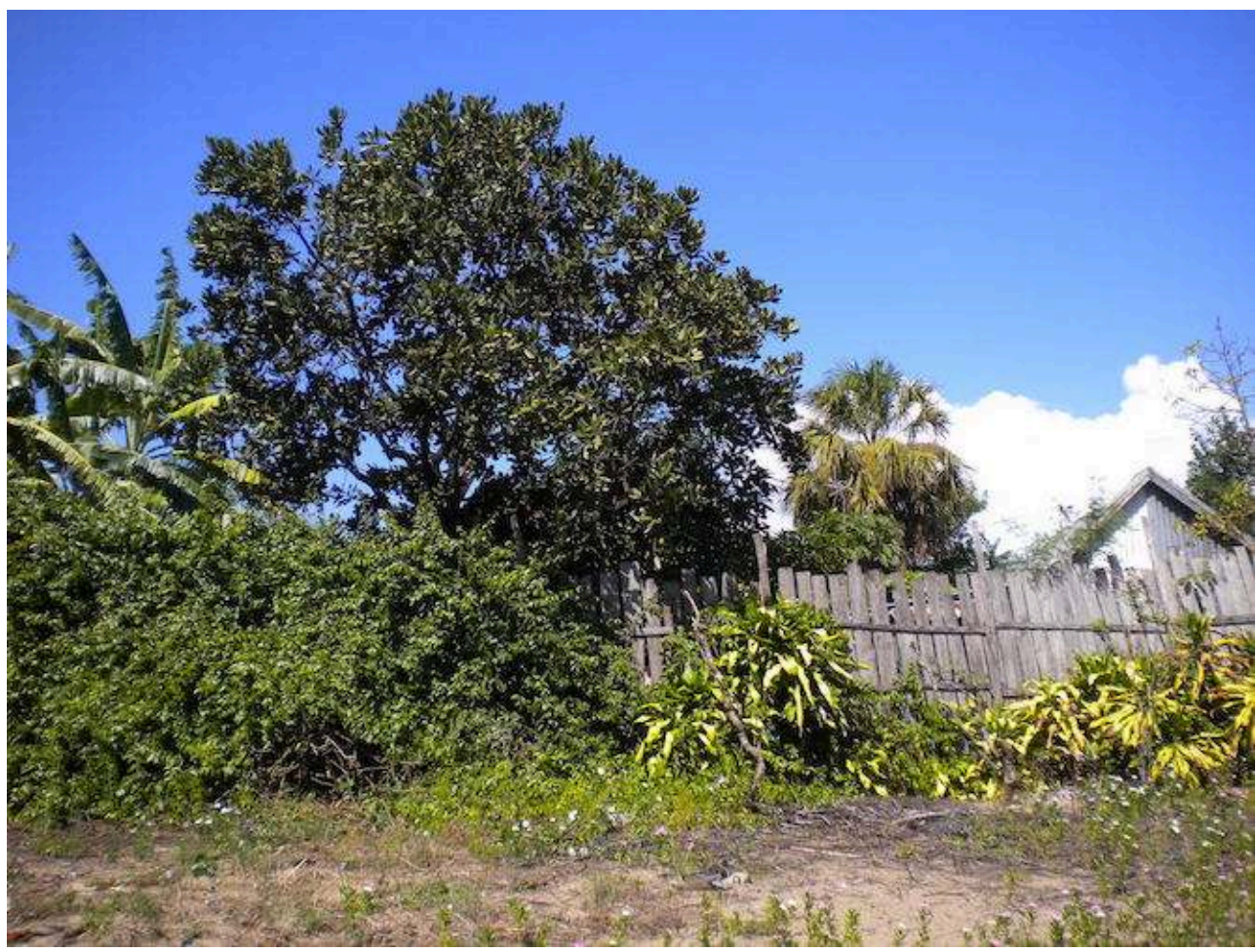

Dans ce quartier encore peu urbanisé, les « concessions » familiales peuvent encore être relativement vastes.

Source : Jean-Baptiste Bing, janvier 2011.

Nourriciers, protecteurs : le vécu rejoint ici l'imaginaire habituellement conféré aux arbres (Corbin, 2013; Harpet, 2014). Cette multifonctionnalité des arbres n'est pas propre à Tanamakoa et Analakinina : outre les parties du centre-ville touchées par une certaine pauvreté, elle caractérise également l'ensemble des quartiers périphériques de Tamatave, qu'ils soient d'urbanisation ancienne (plus près du centre, au parcellaire souvent étroit: figure 9) ou plus récente (parcellaire plus large, généralement plus éloigné du centre : figure 10). Il semble que ladite multifonctionnalité de ces arbres urbains se rapproche de celle qui, en zone rurale, caractérise l'agroforesterie (Torquebiau, 2007; Bing, 2015b). L'analogie paraît d'autant plus forte qu'une importante partie de la population de ces quartiers a connu l'exode rural, que les pratiques paysannes restent présentes dans les mémoires (surtout quand des attaches fortes ont été conservées avec le village, ce qui est souvent le cas même après une ou deux générations), et que les relations socioéconomiques conservent une bonne part des habitudes rurales. S'il paraît abusif de parler au sens fort d' " agroforêt urbaine ", en revanche on peut évoquer le «jardin-forêt » qui est l'une des formes née de pratiques agroforestières compatibles avec un environnement urbain (Torquebiau, 2007, p. 48-54).

À l'appui de cette hypothèse, nous ferons appel à un argument concernant le rôle écologique et sanitaire des arbres en ville. Dans le contexte actuel de crise de l'État et des autorités publiques (qui, à Madagascar, semble remonter aux années 1960: Tiersonnier, 2001 ; Bing, 2012), les infrastructures urbaines sont souvent délabrées : le macadam de certaines artères du centre-ville a fait place à des nids que l'on dirait « de dinosaure " plutôt que "de poule", et les rues et ruelles secondaires n'ont parfois 
jamais été macadamisées. Les habitants les plus pauvres (qu'il s'agisse de ceux squattant les demeures à moitié ruinées d'Ampasimazava ou de ceux qui vivent dans les quartiers d'urbanisme spontané) ont ainsi à faire face à l'alliance du climat hyperhumide et du mauvais entretien des rues et ruelles, qui entraîne régulièrement l'apparition de flaques stagnantes qui peuvent subsister plusieurs semaines, voire, au cœur de la saison des pluies, plusieurs mois (figure 9); cela favorise des parasites variés, dont les moustiques qui transmettent (entre autres) la dengue et le paludisme. Face à cette situation sanitaire désastreuse, les arbres jouent un rôle essentiel : de par leur développement racinaire et leurs besoins en eau, ils contribuent à renforcer les capacités d'absorption du sol et à pomper les excès de pluie (Havlicek, 2016, p. 20-22, p. 20-32). Remarquons d'ailleurs que l'arbre en tant que tel est défini ici par ses fonctions (écologique et sanitaire, donc, mais aussi nourricière et protectrice), donc de manière vernaculaire, pratique et concrète, et non en se fondant sur la classification botanique savante: cette dernière exclurait la vaste famille des palmiers (Arecaceraceae), ces herbes arborescentes qui, nombreuses à Tamatave (cocotiers : Cocos nucifera, figures 4 et 6 ; palmiers-bouteilles : figure 5 ; ravenalas...), fournissent on l'a dit fruits, matières premières, décoration, ombrage et maintien du sol.

La triple dimension de l'agroforesterie, qui fait remplir aux formes qui en sont issues des fonctions économiques (production agricole), sociales (convivialité, mémoire...) et écologiques (régulation hydrologique...), favorise un déploiement de ressources dans un environnement restreint (Torquebiau, 2007, p. 114-115). Cependant, pour parler réellement d'un paysage sylvicole urbain, il faut certes que cette forme fasse "se toucher la terre et le ciel » (expression calquée sur celle de Corajoud, 2010), mais il faut aussi que des représentations (littéraires, iconiques...: Berque, 2008) dudit paysage existent. Or, si jardin et paysage urbain forment deux géogrammes bien identifiés et à la richesse imaginaire déjà largement investie par les artistes francophones et malgachophones, en revanche forêt urbaine et jardin-forêt demeurent moins identifiés en tant que tels (c'est aussi le cas de l'agroforêt dans les campagnes : Bing, 2015b). Ainsi, la ripisylve qui borde le canal de Pangalanes et qui traverse toute la ville est complètement délaissée et ignorée des habitants (figure 11). Pourtant des analogies sont possibles (comme on l'a vu dans le cas de la controverse autour des places d'Ampasimazava) mais (à l'instar de la gentrification des bâtiments en bois) cela demanderait à être confirmé. On peut en tout cas supposer qu'une telle institutionnalisation passerait par la mobilisation des autorités de quartier (fokontany), déjà largement sollicitées pour assurer toute une série de tâches administratives délaissées par les professionnels de la mairie, et par la culture de l'entraide communautaire (fihavanana) qui demeure l'un des principaux remparts face à la décomposition des institutions (Alexandre, 2003). 
Figure 11. Le canal des Pangalanes, à son embouchure entre Analakinina (à gauche) et le centreville (à droite)

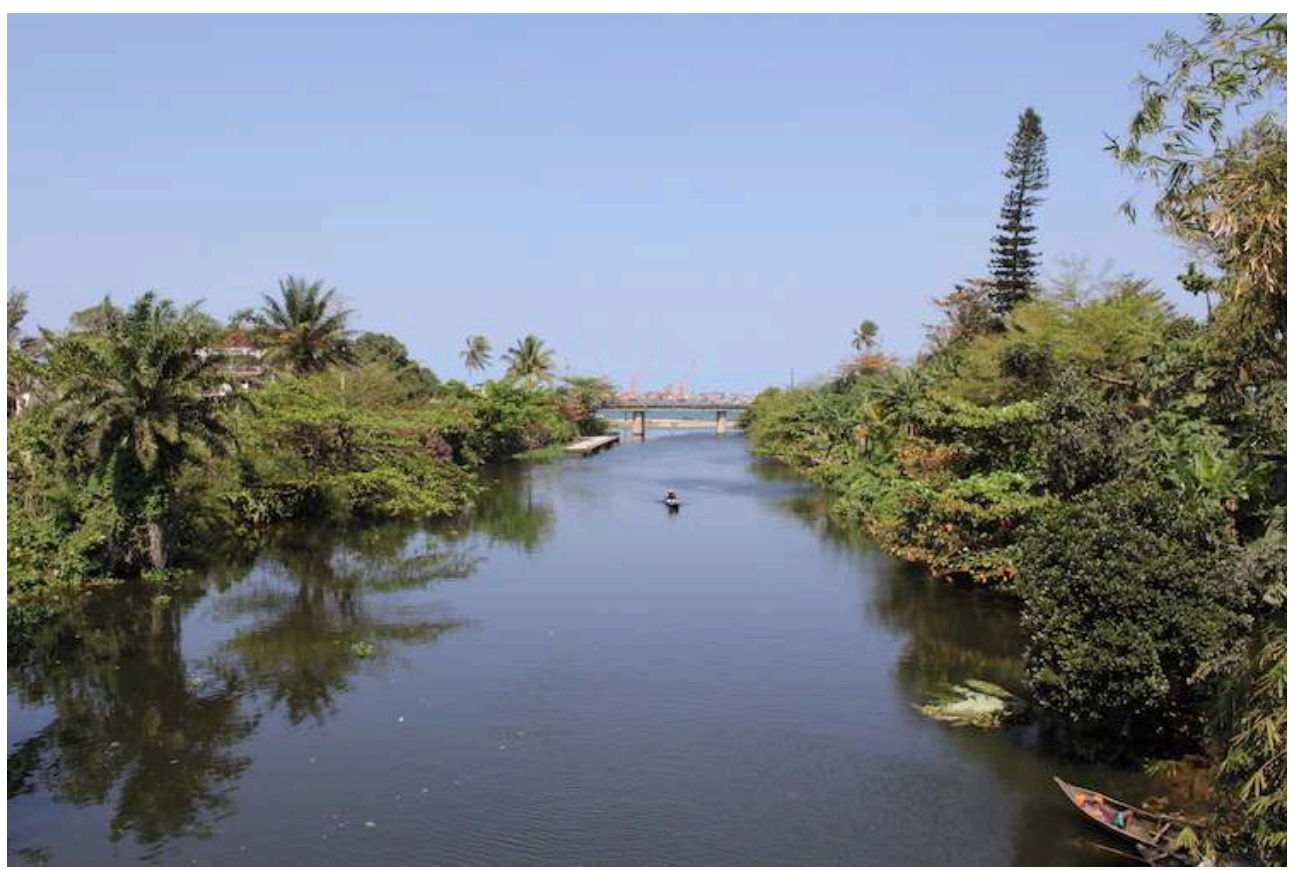

La véritable ripisylve urbaine qui le borde est délaissée à la fois par la municipalité et par les habitants ; véritable dépotoir, elle abrite toute une faune commensale. Il est vrai que, délaissé, le canal lui-même (impraticable à la navigation dans presque toute la ville, sauf sur les quelques centaines de mètres de l'embouchure) l'est tout autant...

Source : Ludovic Pagniez, septembre 2010.

\section{Conclusion}

À la fois inscrit dans la longue durée et dans le quotidien, l'arbre apparaît - à l'instar du paysage - à même de lier en lui et par lui les différentes temporalités qui tissent l'histoire de la ville et les différents niveaux de territorialités qui, par-delà les conflits qui peuvent les opposer, continuent de la faire vivre. Il y a donc plus qu'une simple analogie entre la diversité des arbres tamataviens et le caractère métis du paysage urbain : de par les identités qui s'y expriment, de par les enjeux de pouvoir qui les mobilisent, les arbres contribuent à construire ce paysage urbain métissé, dont la créolité s'exprime surtout à travers le bâti que les arbres (dans l'aménagement des jardins et des rues) contribuent à enrichir. Enfin leurs usages multiples, qui ressortent à la fois de l'esthétique, de la décoration et du symbolique comme de l'écologique, du concret et du pratique dans les quartiers pauvres comme dans les quartiers plus aisés, continuent à faire de cette ville, et notamment des zones délaissées par les autorités, un espace habitable dans des conditions plus ou moins décentes. Alors que, depuis 2014, la situation politique du pays semble en voie de normalisation, il sera intéressant de voir sur le long terme si une municipalité plus stable et disposant de ressources plus assurées saura défendre et exploiter ce patrimoine arboré, et la place qu'elle accordera à la population dans cette gestion. 


\section{BIBLIOGRAPHIE}

Alexandre, C., Violences malgaches, Antananarivo, Foi et justice, 2007, 196 p.

Alexandre, C., Le Malgache n'est pas une île, Antananarivo, Foi et Justice, 2003, 64 p.

Bavoux, C., Islam et Métissage. Des musulmans créolophones à Madagascar. Les Indiens sunnites sourti de Tamatave, Paris, L'Harmattan, 1990, 137 p.

Berque, A., Écoumène. Introduction à l'étude des milieux humains, Paris, Belin, 2009, 447 p.

Berque, A., La Pensée paysagère, Paris, Archibooks, 2008, 107 p.

Bing, J.-B., « Heuristique de la lenteur. Pour une géographie qui soit mésologie vécue », Carnets de géographes, n 8, 2015a, 8 p., URL : http://www.carnetsdegeographes.org/carnets_terrain/ terrain_08_01_Bing.php

Bing J.-B., « Agroforêt : formes et pratiques héritées en Indonésie et à Madagascar », Le Globe, vol. 155, 2015b, p. 89-96, URL : http://www.persee.fr/doc/

globe_0398-3412_2015_num_155_1_7378

Bing, J.-B., « Antenina (Magagascar). Ces "côtiers" qui, de leurs "montagnes", n'ont jamais vu la mer ", Revue de géographie alpine-Journal of Alpine Research, rubrique « Lieux-dits », 2014, URL : http://rga.revues.org/2534

Bing, J.-B., « Du bon usage de la malgachisation. L'écolinguistique de l'école en pays betsimisaraka au regard de la géographie culturelle ", Études Océan Indien, n 48, 2012, 16 p., URL : https://oceanindien.revues.org/1687

Corajoud, M., Le Paysage, c'est l'endroit où le ciel et la terre se touchent, Arles, Actes Sud, 2010, 271 p. Corbin, A., La Douceur de l'ombre. L'arbre source d'émotions de l'Antiquité à nos jours, Paris, Fayard, 2013.

Ernwein, M. et Sgard, A., « "Les quatre mondes du lac Léman" ou explorer avec des non-voyants un paysage polysensoriel », Cahiers de géographie du Québec, vol. 56, n 158, 2012, p. 279-295.

Harpet, C., «Au pied de l'arbre (Ambody Kahazo) : pensée malgache du végétal », dans Harpet, C., Billet, P. et Pierron, J.-P. (dir.), À l'ombre des forêts. Usages, images et imaginaire de la forêt, Paris, L'Harmattan, 2014, p. 195-207.

Havlicek, E., « Le sol urbain : surface inerte ou capital naturel ? ", dans Mantziaras, P. et Viganò, P. (dir.), Le Sol des villes, Genève, MétisPresses, 2016, p. 19-35.

Illich, I., La Convivialité, Paris, Éditions du Seuil, 1973, 169 p.

Men, P., Mangalaza, E et Weiss, É., Tamatave l'irrésistible, Paris, SDE, 2003.

Péguy, C.-P., Espace, temps, complexité. Vers une métagéographie, Paris, Belin, 2001, 283 p.

Raffestin, C., « Langue et territoire. Autour de la géographie culturelle », dans Walty, S. et Werlen, B. (ed.), Kulturen und Raum : theoretische Ansätze und empirische Kulturforschung in Indonesien : Festschrift für Professor Albert Leemann, Zurich, Rüegger, 1995, p. 87-104.

Sansot, P., Variations paysagères. Invitation au paysage, Paris, Klincksieck, 1983, 163 p.

Tiersonnier, J., Les Missionnaires au service du développement, Antananarivo/Paris, Ambozontany/ L'Harmattan, 2001, 218 p. 
Torquebiau, E., L'Agroforesterie. Des arbres et des champs, Paris, L'Harmattan, 2007, 151 p.

Tuan, Y.-F., Espace et Lieu. La perspective de l'expérience, Gollion, Infolio, 2006, 219 p.

\section{NOTES}

1. Pour plus de détails sur la démarche et la méthodologie suivie, $c f$. l'article écrit sur ce sujet à propos de mon autre terrain de recherche, l'Indonésie occidentale (Bing, 2015a). Certes le pays se trouve de l'autre côté de l'océan Indien, mais le rapport établi avec les habitants en est proche et avait d'ailleurs la même origine : le volontariat de solidarité internationale.

2. Si les deux termes ne doivent pas être confondus, chacun recouvre une part de la réalité tamatavienne ou majungienne. Nous choisirons de mettre l'accent moins sur la multiculturalité (coexistence de plusieurs cultures en un temps T et en un lieu L) que sur le métissage (processus de mélange d'éléments issus de cultures multiples, qui en retour influence celles-ci) qui, sur la longue durée, en a résulté. Signalons de plus que la créolisation est une forme particulière de métissage, qui débouche sur l'élaboration d'une culture originale nouvelle.

3. Le terme et les réalités qu'il recouvre demanderaient à être discutés (cf. Bing, 2014); nous l'emploierons cependant par commodité.

4. Descendants de migrants venus durant la période coloniale, ils ont généralement pris la nationalité malgache à l'Indépendance. Alors que les métis sino-malgaches ne sont pas rares et que les Sinoa ("Chinois») sont souvent bien intégrés, les Karanes (Indo-malgaches) demeurent presque toujours considérés comme des allochtones auxquels on reproche de former des communautés fermées sur elles-mêmes. À ce sujet, $c f$. Bavoux (1990, p. 8-30).

5. Presque tous des étrangers expatriés. Les rares zanatany (" enfants de la Terre ", descendants de colons ayant fait souche sur place et ayant pris la nationalité malgache) sont parfois confondus avec eux.

6. Comme toute l'analyse de cet article, il ne s'agit pas ici de prétendre fournir des éléments fondés sur une étude statistique ; il s'agit plutôt d'un bilan global qui ressort après deux années de discussions et d'insertion dans la société locale...

7. «Enfants de la terre ». L'expression est très ambiguë, puisqu'elle peut désigner à la fois les autochtones par opposition aux autres Malgaches (à Tamatave, les Betsimisaraka par opposition aux Merina, aux Bezanozano ou aux Antandroy) et les étrangers et descendants d'étrangers considérés comme intégrés.

8. Ce terme, ici et dans l'article, est à entendre en son sens berquien de " prise médiale » : il s'agit d'un élément de l'environnement qui, à la fois, porte du sens et offre une possibilité d'agir sur ledit environnement.

9. D'autres bâtiments et éléments du mobilier urbain, plus rares, expriment diverses influences : pagode de la "congrégation chinoise ", styles arabisant des mosquées et européen des églises, monumentalité de type soviétique de la Banque centrale malgache, bancs art nouveau en front de mer, maisons basques près du boulevard de la Bourdonnais...

10. Mais pas seulement, notamment à Ampasimazava : la communauté sourti, fort appauvrie, en occupe une partie; de plus de nombreux bâtiments abandonnés, plus ou moins en ruine, parsèment tout le centre - $\mathrm{y}$ compris à deux pas de maisons bien plus luxueuses : les quelques dizaines de mètres qui séparent le consulat britannique de la place Bienaimé, par exemple, offrent un spectacle de ce genre.

11. D'abord champ de course, elle fut transformée en axe reliant le port à la gare ; puis l'hôtel de ville fut construit devant la gare, amputant légèrement l'avenue. Enfin, depuis l'Indépendance, les chemins de fer (et la gare de Tamatave) ont été quasiment abandonnés. 
12. La croissance de la ville n'ayant jamais cessé, les actuels cimetières et marchés sont à nouveau complètement enclavés et trop petits; de nouveaux lieux dédiés à ces fonctions, ouverts en périphérie, viennent donc compléter les anciens, dont l'activité se poursuit.

13. Sport dont les Malgaches sont férus, et où ils occupent une place élevée dans la hiérarchie mondiale. Que ce sport typiquement français ait été complètement acculturé et se pratique sur une ancienne place sacrée betsimisaraka couverte d'arbres importés d'Inde est un bon exemple du métissage tamatavien.

14. Crâne d'un zébu sacrifié rituellement, et planté en fin de cérémonie. À une quinzaine de kilomètres au nord de la ville, l'entrée d'une station d'essai forestière (d'origine coloniale elle aussi) a également été plantée de banians. L'espace, vaste, sert parfois de lieu de rassemblement ou de fête ; cependant des bucranes ont été implantés soit au cœur de la station, soit dans des espaces villageois. Le lieu en question, malgré ses banians, n'a donc pas été sacralisé.

15. Et témoigne d'une confusion entre les différents pays d'Extrême-Orient que mon épouse, Indonésienne, a souvent constatée. Elle ne manquait d'ailleurs jamais de rectifier l'appellation "litchi chinois", précisant que rambutan signifiait "fruit à cheveux" (du malais rambut, " cheveu », ce qui est effectivement plus adapté : le fruit en question ressemble en effet à un litchi doté de dreadlocks...).

\section{RÉSUMÉS}

Deuxième plus grosse agglomération et premier port de Madagascar, Tamatave doit à son rôle d'interface entre l'île et l'extérieur un caractère métis. Cet article interroge la place et les usages de l'arbre dans la construction de ce métissage paysager urbain. S'appuyant sur deux années de séjour sur place ayant permis de croiser les méthodes (parcours commenté, flânerie urbaine, entretiens formels et informels, observation participative...), il analyse essentiellement trois quartiers de la ville (Tanamakoa, Analakinina et le centre-ville). La première partie dégage les éléments théoriques et historiques qui précisent le lien du paysage urbain tamatavien au métissage et à la créolité de la ville; la deuxième examine la pluralité des fonctions de l'arbre dans ce paysage, les rattache aux différentes territorialités qui s'y expriment et aboutit à quelques pistes de réflexion en matière de pluridimensionnalité du paysage de la ville, de sa gestion et de ses évolutions.

The second-largest agglomeration and first port of Madagascar, Toamasina, owes its position as the interface between the island and the outside world to its cross-cultural background. This article investigates the place occupied by and the uses made of trees in this mixed urban landscape. Based on two years spent in the city comparing methods (guided tours, urban strolls, formal and informal interviews and participative observations) the article investigates three areas in the city (Tanamakoa, Analakinina and the city centre). The first part of the article presents the theoretical and historical background revealing the links between the urban landscape of Toamasina and the mixed Creole identity of the city; the second examines the plural nature of the functions of the tree in this landscape, relating these to the different territorial dimensions expressed through the trees, and finally outlines further aspects for consideration regarding the pluridisciplinary nature of the urban landscape, its management and its evolutions. 
INDEX

Mots-clés : sylviculture urbaine, agroforesterie, urbanisme, métissage, géographie

Keywords : urban forestry, agroforestry, urbanism, cross-cultural influence, geography

\section{AUTEUR}

\section{JEAN-BAPTISTE BING}

Jean-Baptiste Bing est doctorant à l'université de Genève, faculté des sciences de la société, département de géographie et environnement. Sa thèse porte sur les savoirs agroforestiers à Java, dans le Sud de Sumatra et dans l'Est malgache. Il a également rédigé une dizaine d'articles concernant les divers médias (cinéma, conte, langue...) et manifestations (protection de l'environnement, patrimonialisation...) prises par les interrogations qui traversent les sociétés malgache et indonésienne à propos de la modernité.

jean-baptiste.bing[at]unige[dot]ch 